\title{
Thrombospondin-1 protects against pathogen-induced lung injury by limiting extracellular matrix proteolysis
}

\author{
Yanyan Qu, ${ }^{1}$ Tolani Olonisakin, ${ }^{1}$ William Bain, ${ }^{1}$ Jill Zupetic, ${ }^{1}$ Rebecca Brown, ${ }^{1}$ Mei Hulver, ${ }^{1}$ \\ Zeyu Xiong, ${ }^{1}$ Jesus Tejero, ${ }^{1,9}$ Robert M.Q. Shanks, ${ }^{2,3}$ Jennifer M. Bomberger, ${ }^{3}$ Vaughn S. Cooper, ${ }^{3}$ \\ Michael E. Zegans, ${ }^{4}$ Hyunryul Ryu, ${ }^{5}$ Jongyoon Han, ${ }^{5,6,7}$ Joseph Pilewski, ${ }^{1}$ Anuradha Ray, \\ Zhenyu Cheng, ${ }^{8}$ Prabir Ray, ${ }^{1}$ and Janet S. Lee ${ }^{1,9}$ \\ 'Division of Pulmonary, Allergy, and Critical Care Medicine, Department of Medicine, ²Department of Ophthalmology, \\ and ${ }^{3}$ Department of Microbiology and Molecular Genetics, University of Pittsburgh, Pittsburgh, Pennsylvania, USA. \\ ${ }^{4}$ Department of Microbiology and Immunology, Dartmouth Geisel School of Medicine, Hanover, New Hampshire, USA. \\ ${ }^{5}$ Research Laboratory of Electronics, ${ }^{6}$ Department of Electrical Engineering and Computer Science, and ${ }^{7}$ Department \\ of Biological Engineering, Massachusetts Institute of Technology, Cambridge, Massachusetts, USA. ${ }^{8}$ Department of \\ Microbiology and Immunology, Dalhousie University, Halifax, Nova Scotia, Canada. ${ }^{9}$ Vascular Medicine Institute, University \\ of Pittsburgh, Pittsburgh, Pennsylvania, USA.
}

Acute lung injury is characterized by excessive extracellular matrix proteolysis and neutrophilic inflammation. A major risk factor for lung injury is bacterial pneumonia. However, host factors that protect against pathogen-induced and host-sustained proteolytic injury following infection are poorly understood. Pseudomonas aeruginosa (PA) is a major cause of nosocomial pneumonia and secretes proteases to amplify tissue injury. We show that thrombospondin-1 (TSP-1), a matricellular glycoprotein released during inflammation, dose-dependently inhibits PA metalloendoprotease LasB, a virulence factor. TSP-1-deficient (Thbs 1/-) $^{- \text {}}$ mice show reduced survival, impaired host defense, and increased lung permeability with exaggerated neutrophil activation following acute intrapulmonary PA infection. Administration of TSP-1 from platelets corrects the impaired host defense and aberrant injury in Thbs 1/- $^{-1}$ mice. Although TSP-1 is cleaved into 2 fragments by PA, TSP-1 substantially inhibits Pseudomonas elastolytic activity. Administration of LasB inhibitor, genetic disabling of the PA type II secretion system, or functional deletion of LasB improves host defense and neutrophilic inflammation in mice. Moreover, TSP-1 provides an additional line of defense by directly subduing host-derived proteolysis, with dose-dependent inhibition of neutrophil elastase from airway neutrophils of mechanically ventilated critically ill patients. Thus, a host matricellular protein provides dual levels of protection against pathogen-initiated and hostsustained proteolytic injury following microbial trigger.

Conflict of interest: The authors have declared that no conflict of interest exists.

Submitted: August 21, 2017

Accepted: December 27, 2017

Published: February 8, 2018

Reference information: JCI Insight. 2018;3(3):e96914. https:// doi.org/10.1172/jii.insight.96914.

\section{Introduction}

Bacterial pneumonia is a common risk factor for the development of acute lung injury, or the acute respiratory distress syndrome (ARDS) as it is clinically known, which is often characterized by unbridled airspace neutrophilic inflammation and the final common pathway for a variety of direct and indirect insults to the lungs. Bacterial pneumonia is also a common complication of ARDS and one that contributes to substantial ARDS morbidity including prolonged length of mechanical ventilation, hospitalization, and increased economic burden due to persistent lung inflammation and injury (1-5). Pseudomonas aeruginosa is a nonfermenting Gram-negative bacillus and opportunistic pathogen that is a common cause of hospital-associated acute lower respiratory tract infections, and a common complication of ARDS (6). The pathogen plagues a significant number of critically ill patients (1), the immunocompromised (7), and the elderly $(8,9)$. Out of all organisms identified in a prospective, international collaborative study of sepsis epidemiology in multiple intensive care units (ICUs), $P$. aeruginosa is the only pathogen found to be independently associated with increased 60-day mortality (10).

$P$. aeruginosa secretes a broad arsenal of cell-associated and extracellular virulence factors, such as lipopolysaccharide, flagellin, proteases, exotoxins, pyocyanin, siderophores, hemolysins, and phospholipases 
$(11,12)$. These virulence factors are toxinogenic, invasive, and implicated in airway and lung parenchymal damage. Among the virulence factors secreted by $P$. aeruginosa, the protease LasB, otherwise known as Pseudomonas elastase or pseudolysin, is a secreted type II $\mathrm{Zn}^{2+}$ metalloendopeptidase that degrades tissue elastin and collagen, presumably to liberate nutrients for pathogen growth in addition to degrading host defense molecules such as surfactant protein A and D, complement, and mucins $(13,14)$. A study of nonclonal $P$. aeruginosa strains isolated from critically ill patients with ventilator-associated pneumonia suggests that elastase production is one of the most important virulence factors associated with increased lung injury when tested in mice (15). Yet, the role of host-derived factors that protect against pathogen-triggered proteolytic lung injury is not fully known.

Thrombospondin-1 (TSP-1) is a calcium-binding, matricellular glycoprotein implicated in important biological functions such as embryogenesis, wound repair, tumor growth and metastasis, angiogenesis, hemostasis, and inflammation (16-21). TSP-1 binds multiple ligands in vitro (21), but the functional consequences of TSP-1 binding to the majority of these ligands and the net effect of these interactions under different physiologic contexts have yet to be realized. Platelet $\alpha$-granules are a major reservoir of TSP-1 in circulation and TSP-1 is released during platelet activation $(19,22)$. Platelet proteins such as TSP-1 are found in the airspaces of ARDS patients and TSP-1 concentrations correlate with composite injury scores that quantify the degree of lung injury (23). Although platelets are a major source of TSP-1 in vivo, other cells such as endothelial $(24)$ and myeloid cells $(25,26)$ also express TSP-1 at specific sites of inflammation and injury. Indeed, others have shown that pulmonary endothelium-derived TSP-1 or TSP-1 purified from activated platelets can drive bronchioloalveolar stem cell differentiation into alveolar type II cells following bleomycin-induced lung injury to facilitate repair (24).

We previously showed that TSP-1 is an endogenous, host protective molecule that counters inflammation by triggering antiinflammatory cytokine IL-10 production in macrophages during the resolution phase of experimental lipopolysaccharide-induced (LPS-induced) lung injury (27). We further showed that TSP-1 curtails neutrophil granule serine protease activity of neutrophil elastase (NE) and cathepsin $\mathrm{G}$ to regulate innate immune response against Klebsiella pneumoniae infection, where unbridled NE activity in TSP-1-deficient

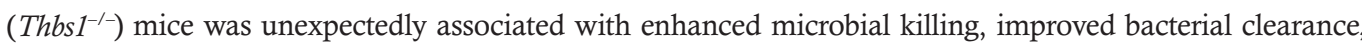
reduced dissemination, and early termination of the inflammatory response (28). While neutrophil serine proteases such as NE contribute to pulmonary host defense in certain situations, mechanisms to curtail the neutrophil protease arsenal are required to prevent an overly vigorous host inflammatory response and subsequent tissue injury. Indeed, excessive neutrophil serine proteases such as NE are found in the airspaces of patients with $\operatorname{ARDS}(29,30)$ and ventilator-associated pneumonia (31). Proteases may, however, be exploited by pathogens such as $P$. aeruginosa to gain entry within the lungs and coopt the host neutrophil arsenal to amplify tissue injury and divert host immune defense. An effective strategy that disarms pathogen-secreted proteases should, therefore, be host protective by limiting pathogen-mediated tissue damage and secondary triggers of aberrant host responses. Given the unique tactics employed by the opportunistic pathogen P. aeruginosa to subvert host immunity and mediate inflammation and injury, we examined the contribution of TSP-1 in the host response to $P$. aeruginosa-mediated injury during acute lower respiratory tract infection.

\section{Results}

TSP-1 deficiency leads to reduced survival, inability to regain weight loss, impaired host defense, and exaggerated inflammatory injury following acute intrapulmonary infection with $P$. aeruginosa. To test the role that TSP-1 plays during $P$. aeruginosa acute intrapulmonary infection, we infected both WT and Thbs $1^{-1-}$ mice with PA14, a reference clinical $P$. aeruginosa strain originally isolated from a burn patient (32). Thbs $1^{-1-}$ mice show reduced survival compared with WT mice (Figure 1A). In those animals that survive the initial infection, Thbs $1^{-1-}$ mice show a failure to regain weight, whereas WT mice fully regain their weights (Figure $1, \mathrm{~A}$

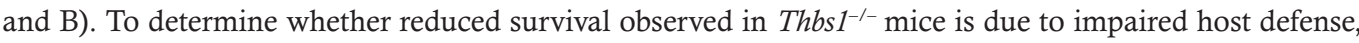
we evaluated lung bacterial burden and inflammatory parameters. As the initial mortality occurred early in Thbs $1^{-/-}$mice, we examined mice prior to this time point and show higher lung bacterial burden, and increased splenic dissemination at 20 hours after infection (Figure 1, C and D). Impaired host defense is accompanied by increased neutrophil recruitment to the lungs, as evidenced by increased total bronchoalveolar lavage (BAL) cells and BAL PMN recruitment (Figure 1, E and F). Thbs $1^{-1-}$ mice also exhibit increased total BAL protein concentrations, a marker of lung microvascular permeability, and increased neutrophil activation as measured by BAL free NE activity (Figure 1, G and H). H\&E staining reveals 
A
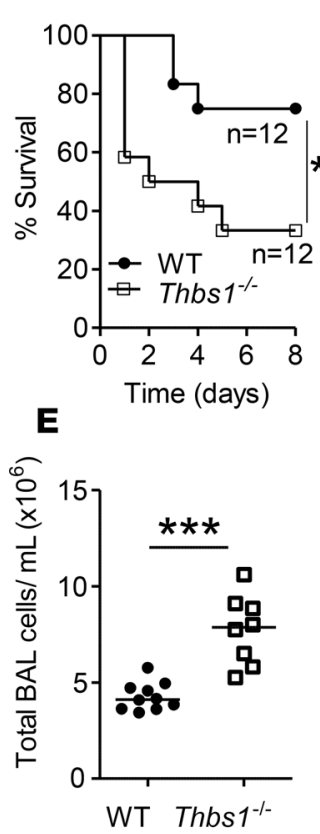

B

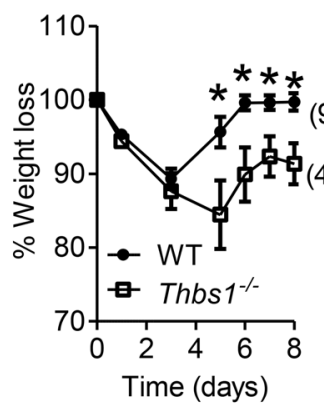

$\mathbf{F}$

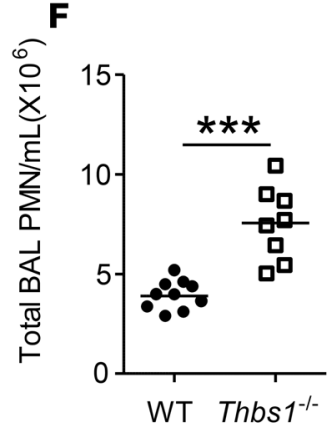

C

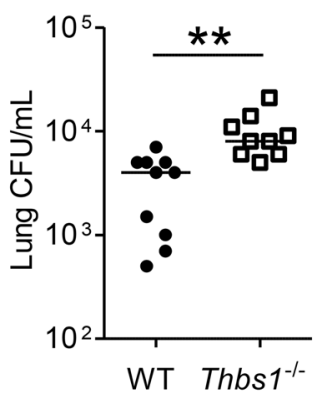

G

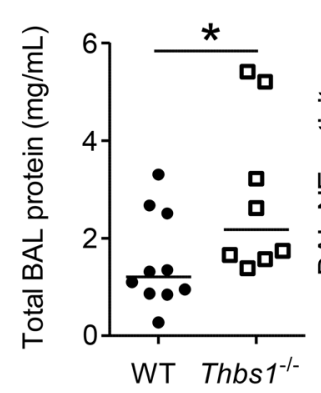

D

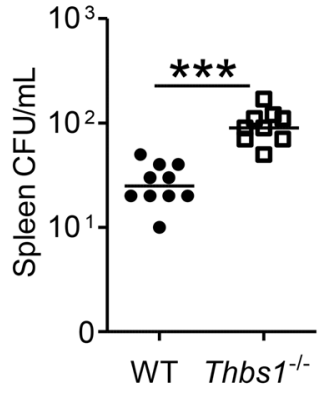

H

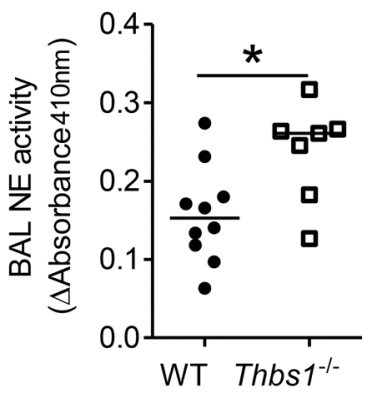

I

WT Oh

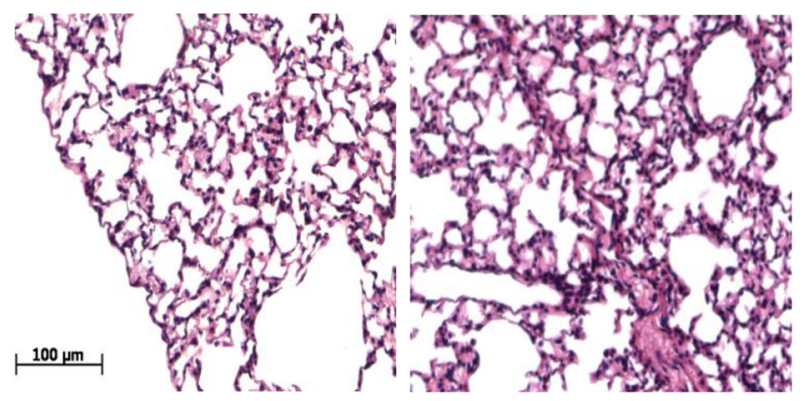

Thbs $1 \%$ oh

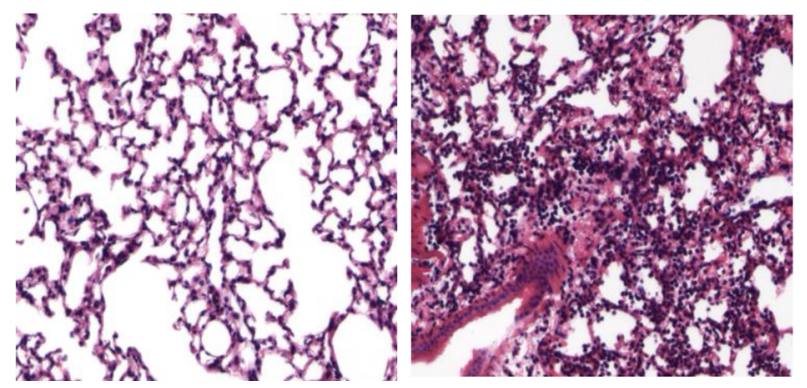

Figure 1. Thbs1/- mice show enhanced mortality, failure to regain weight, higher lung bacterial burden, increased splenic dissemination, and increased airspace neutrophil recruitment and lung microvascular permeability following intratracheal Pseudomonas aeruginosa inoculation. Thbs $1^{-/-}$and WT mice were intratracheally instilled with $P$. aeruginosa (PA) at $10^{6}$ inoculum. (A) Kaplan-Meier survival curve of WT and Thbs $1^{-1-}$ mice following i.t. infection with PA ( $n=12$ mice per group), ${ }^{*} P<0.05$ by log-rank (Mantel-Cox) test. (B) Mice weights were measured for 8 days in WT and Thbs $1^{1-1-}$ mice following i.t. infection with PA ( $n=12$ mice per group), ${ }^{*} P<0.05$ by 2-way ANOVA with Sidak multiple comparisons. (C-H) In separate experiments, mice were sacrificed at a predetermined time point of 20 hours after infection and (C) lung CFU/ml, (D) spleen CFU/ml, (E) total bronchoalveolar lavage (BAL) cell counts/ml, (F) total BAL PMN counts/ml, (C) total BAL protein concentrations, and (H) BAL free neutrophil elastase (NE) activity were measured. (I) Representative H\&E-stained sections of mouse lungs following i.t. PA inoculation. Each data point represents an individual mouse, with lines that indicate the median. The combined results of 2 independent experiments are shown in $\mathbf{C}-\mathbf{H} .{ }^{*} P<0.05,{ }^{* *} P<0.01,{ }^{* *} P<0.001$ by 2 -tailed Mann-Whitney $U$ test.

severe lung tissue inflammation induced by $P$. aeruginosa infection in Thbs $1^{-1-}$ mice when compared with WT mice (Figure 1I). Although WT and Thbs $1^{-/-}$mice show no appreciable differences in basal concentrations of lung cytokines measured, Thbs $1^{-/-}$mice exhibit exaggerated proinflammatory cytokine response with increased lung tissue concentrations of G-CSF, GM-CSF, keratinocyte chemoattractant (KC), IL-1, IL-6, IL-17A, but not IL-10 or TNF- $\alpha$ when compared with WT mice following infection (Figure 2, A-H). These findings suggest that TSP-1 enhances host defense against $P$. aeruginosa infection and curtails the accompanying tissue injury response to microbial trigger.

TSP-1 administration improves antimicrobial host defense and subdues exaggerated inflammatory response in Thbs $1^{-1-}$ mice. To specifically determine whether administration of TSP-1 corrects the defect observed in 
A

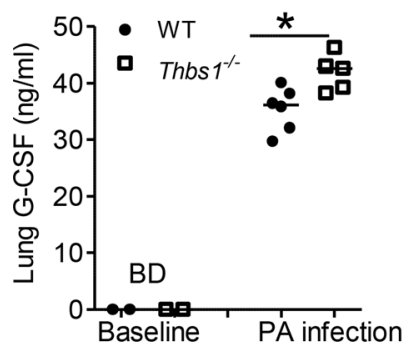

E

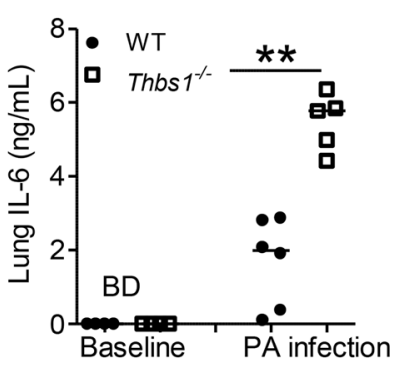

B

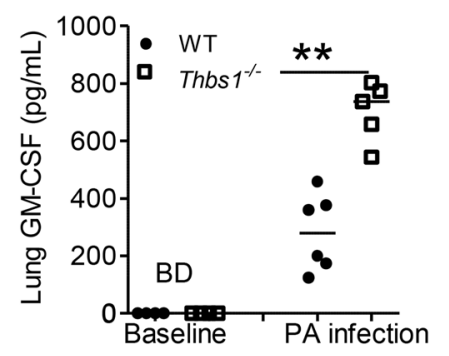

$\mathbf{F}$

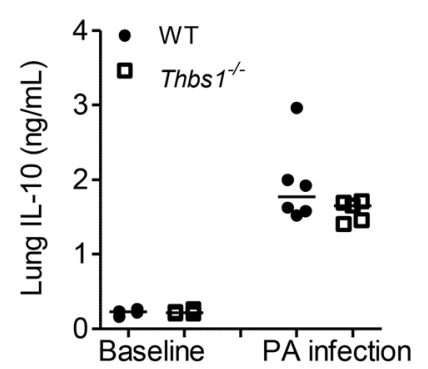

C

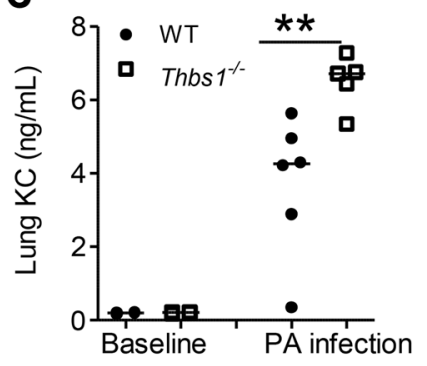

G

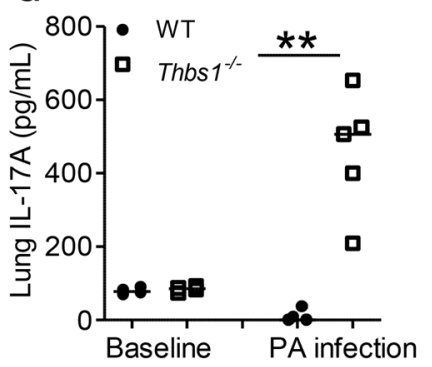

D

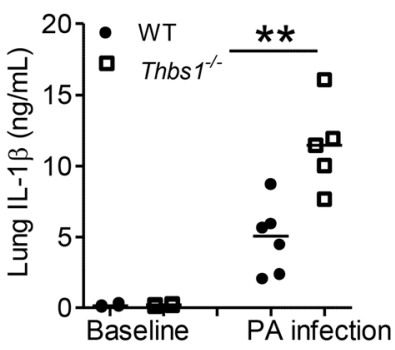

H

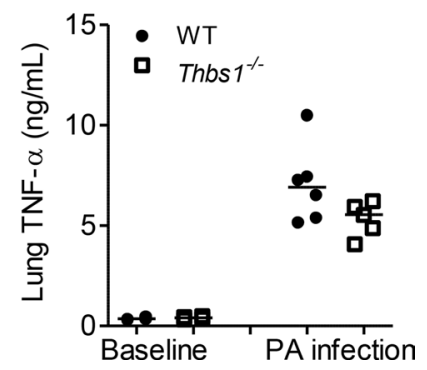

Figure 2. Thbs1/- mice show enhanced lung inflammatory cytokine production following intratracheal Pseudomonas aeruginosa inoculation. Baseline

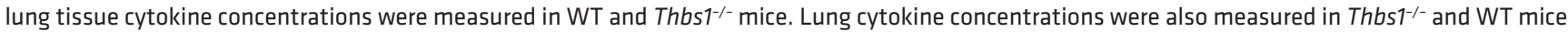
following $P$. aeruginosa (PA) inoculation 20 hours after infection at $10^{6}$ inoculum. (A) G-CSF, (B) GM-CSF, (C) keratinocyte chemoattractant (KC), (D) IL-1 1 , (E) IL-6, (F) IL-10, (C) IL-17A, and (H) TNF- $\alpha$ concentrations were measured in lung tissue homogenates of WT and Thbs $1^{-/-}$mice. Each data point represents an individual mouse, $n=4$ mice per group at baseline and $n=6$ mice per group at 20 hours after infection (one mouse in Thbs ${ }^{1--}$ died prior to tissue harvesting at the 20 -hour time point). $\mathrm{BD}$, below limit of detection. Lines indicate the median. ${ }^{*} P<0.05,{ }^{* *} P<0.01$ by 2 -tailed Mann-Whitney $U$ test.

Thbs $1^{-1-}$ mice during $P$. aeruginosa infection, we tested whether TSP-1 purified from platelets restores host defense and mitigates inflammatory responses in Thbs $1^{-/-}$mice after $P$. aeruginosa infection (24). Administration of TSP-1 reduces lung bacterial burden, total BAL cell counts, and BAL PMN counts in Thbs $1^{-1-}$ mice when compared with vehicle-treated mice (Figure 3, A-C). Lung myeloperoxidase (MPO) activity, a gross measure of total lung tissue neutrophil content, as well as lung tissue NE activity are also reduced in Thbs $1^{-/-}$mice administered TSP-1 compared with Thbs $1^{-/-}$mice administered vehicle (Figure 3, D and E).

On a per-mass basis, TSP- 1 is the most prevalent protein in platelet $\alpha$-granules (33). As platelet releasates (PRs) obtained from WT mice are enriched in TSP-1 in contrast to the absence of TSP-1 in PRs isolated from Thbs $1^{--}$mice (Figure 4A), we also determined whether PRs from WT mice could restore host defense and mitigate inflammatory responses in Thbs $1^{-/-}$mice after $P$. aeruginosa infection. Thbs $1^{-/-}$mice administered WT PRs show reduced lung bacterial burden, and lower BAL total cell and PMN counts when compared with Thbs $1^{1^{--}}$mice administered Thbs $1^{-/-}$PRs (Figure 4, B-D). In addition, lung MPO and lung NE activity are reduced in Thbs $1^{1_{-}^{-}}$mice administered WT PRs compared with Thbs $1^{1^{--}}$mice administered Thbs $1^{1^{--}}$PRs (Figure 4, E and F). Thbs $1^{-/-}$mice administered WT PRs also show reduced cytokine concentrations of GM-CSF, IL-6, and IL-17A, but not G-CSF, KC, IL-10, and TNF- $\alpha$ in lung tissue homogenates compared with those mice administered Thbs $1^{---}$PRs (Figure 4, G-M). Thus, regardless of whether purified TSP-1 or PRs enriched in TSP-1 was utilized, administration of exogenous TSP-1 specifically corrects the impaired host defense and aberrant host injury response observed in Thbs $1^{-1-}$ mice.

The major exoprotease activity secreted by $P A 14$ in vitro exhibits the biochemical profile of the metalloprotease LasB and TSP-1 dose-dependently inhibits LasB protease activity. To investigate the mechanism through which TSP-1 tempers the host response to $P$. aeruginosa infection, we sought to examine whether proteases secreted by $P$. aeruginosa are influenced by TSP-1. First, we sought to identify the dominant class of proteases secreted by PA14. Utilizing an in vitro casein cleavage assay as a screening tool to measure exoprotease activity, we show that PA14 supernatant possesses significant proteolytic activity with the majority inhibited by EDTA, a metalloprotease inhibitor, whereas K. pneumoniae, another clinically relevant extracellular Gram-negative 
A
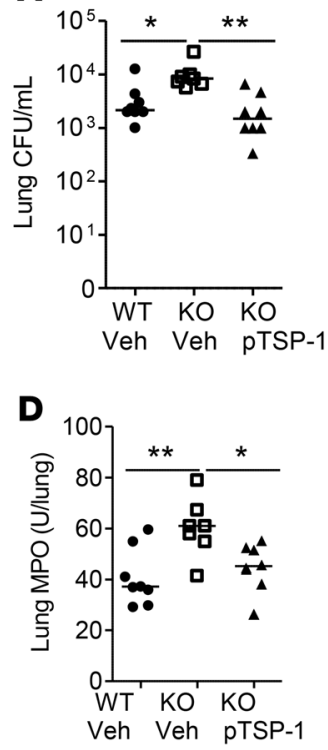

B

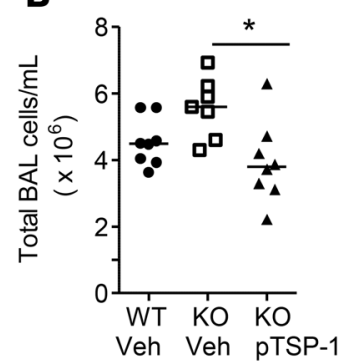

E

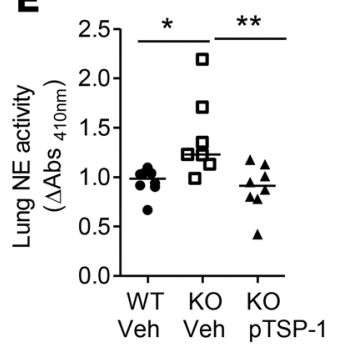

C

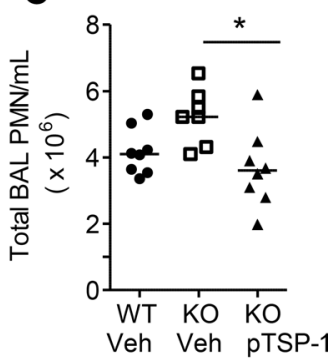

Figure 3. Administration of purified TSP-1 reduced lung bacterial burden, airspace neutrophil recruitment, lung MPO, and lung NE activity in Thbs1/- mice when compared with Thbs $^{-1-}$ vehicle-treated mice. WT and Thbs $1^{-1-}$ mice (KO) were inoculated with Pseudomonas aeruginosa strain PA14 intratracheally at $10^{6}$ inoculum and subsequently administered either vehicle or purified TSP-1 (pTSP-1) by i.p. injection. Outcome measurements were obtained 20 hours after infection. (A) Lung CFU/ml, (B) total bronchoalveolar lavage (BAL) cells counts $/ \mathrm{ml}$, (C) total BAL PMN counts/ml, (D) lung myeloperoxidase (MPO) activity, and (E) lung neutrophil elastase (NE) activity were

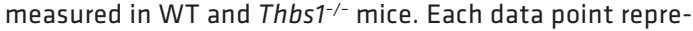
sents an individual mouse, $n=7$ or 8 mice per group. Lines indicate the median. ${ }^{*} P<0.05,{ }^{*} P<0.01$ by KruskalWallis test with Dunn's multiple comparisons.

bacterium, secretes negligible amounts of metalloprotease (Figure 5A). E-64, a cysteine protease inhibitor, fails to dose-dependently inhibit $P$. aeruginosa proteolytic activity and aprotinin, a serine protease inhibitor, inhibits casein cleavage at only higher molar concentrations. Thus, the in vitro protease screen suggests that the major class of proteases secreted by PA14 is metalloprotease.

As the biochemical profile indicated the dominant existence of metalloproteases in the supernatant, we tested whether $N$-mercaptoacetyl-Phe-Tyr-amide (LasBI), a novel dipeptide inhibitor of LasB with a reported $\mathrm{IC}_{50}$ of approximately $41 \mathrm{nM}$ against biofilm formation in vitro (13), blocks PA14 proteolytic activity. LasBI dose-dependently inhibits PA14 proteolytic activity (Figure 5B), suggesting that LasB accounts for the majority of secreted protease activity by PA14. Moreover, LasBI attenuates the ability of $P$. aeruginosa supernatant to cleave the specific LasB substrate aminobenzoyl-Ala-Gly-Leu-Ala- $p$-nitrobenzyl-amide (Figure 5C) and purified LasB to cleave elastin in a dose-dependent manner (Figure 5D). Further, recombinant human TSP-1 (rhTSP-1) inhibits the ability of PA14 supernatant to cleave casein in a dose-dependent fashion (Figure 5B). Finally, purified TSP-1 from human platelets dose-dependently inhibits $P$. aeruginosa LasB activity (Figure 5C) and that human PR enriched in TSP-1 restrains the ability of LasB to cleave elastin over time (Figure 5D). It is worth noting that neither TSP-1 nor LasBI show direct antimicrobial activity against PA14 growth in vitro (Supplemental Figure 1; supplemental material available online with this article; https://doi.org/10.1172/jci.insight.96914DS1). Thus, our data indicate that TSP-1 is a host-derived matricellular protein that potently inhibits pathogen-derived exoprotease LasB activity in vitro but does not exhibit direct antimicrobial effects.

TSP-1 is cleaved by P. aeruginosa but TSP-1 displays inhibitory activity against P. aeruginosa elastase in vitro. LasB degrades host defense molecules such as the antimicrobial peptide cathelicidin and surfactant protein $\mathrm{D}$, and inactivates endogenous NE inhibitors such as elafin and secretory leukocyte protease inhibitor (SLPI) in vitro $(34,35)$. However, the host protective mechanisms that persist during pathogen-induced injury is less well known. We tested whether $P$. aeruginosa-secreted proteases can proteolytically cleave TSP-1. It was previously shown that NE cleaves TSP-1 N-terminal to the disulfide-bonded knot, separating the heparin binding domain from the rest of the molecule $(36,37)$. However, this cleavage does not prevent TSP-1 inhibitory function against NE because the TSP-1 type III repeats (TSR3) region located near the C-terminus remains intact $(36,37)$. We show that the supernatant from WT PA14 cleaves TSP-1 into 2 fragments represented by approximately $30-\mathrm{kDa}$ and approximately $130-\mathrm{kDa}$ bands (Figure $6 \mathrm{~A}$ ), similarly to the cleavage pattern caused by NE. In contrast, the supernatant from $K$. pneumoniae, which lacks protease activity or PA14lasB::Tn5 (a PA14 transposon insertion mutant with deficient protease, elastase, and specific LasB activity) (Supplemental Figure 2) fails to cleave TSP-1. However, TSP-1 displays potent inhibitory function against $P$. aeruginosa elastase activity over the same time period (Figure 6B). Taken together, these data suggest that pathogen-derived exoprotease cleaves the host protein TSP-1, but TSP-1 inhibitory activity against LasB remains intact in vitro. 
A

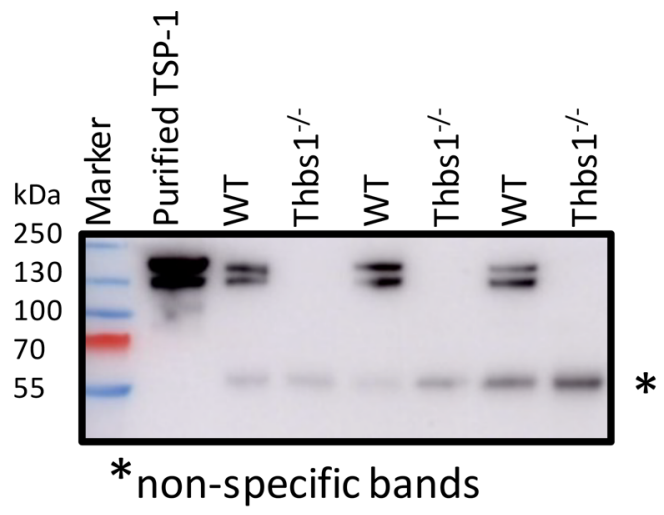

B

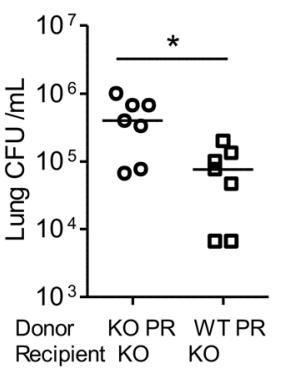

$\mathbf{F}$

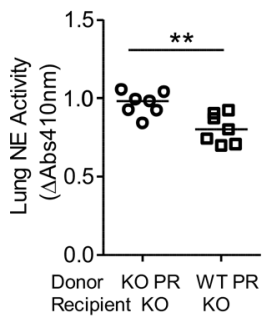

J

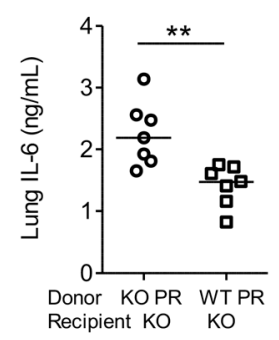

D

\section{E}

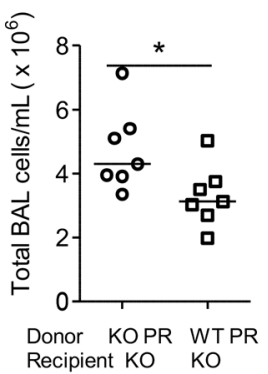

G
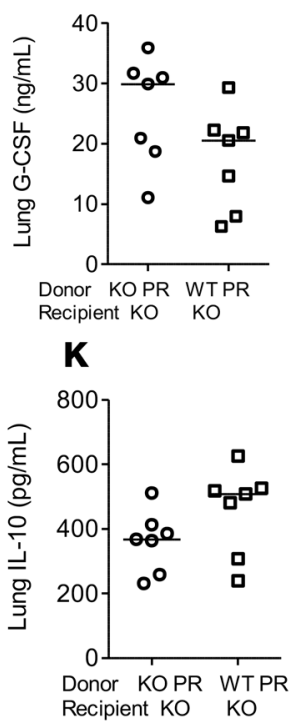
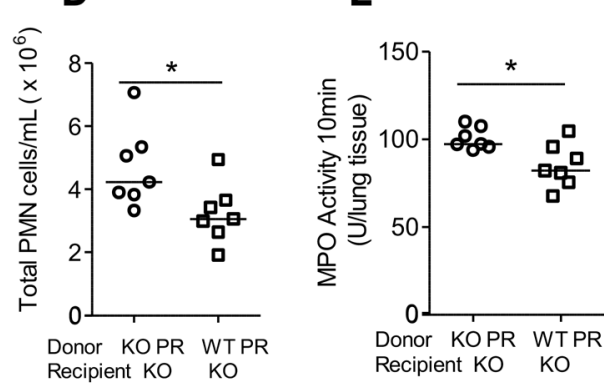

H
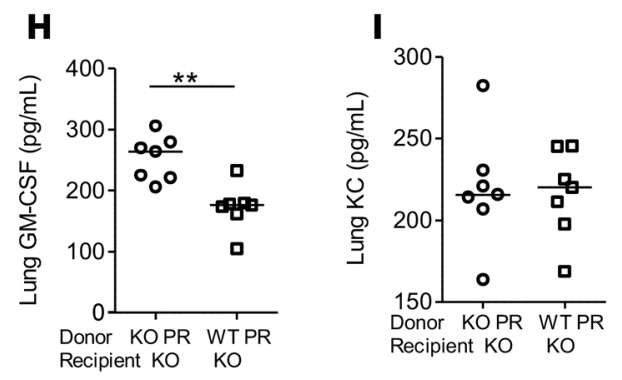

$\mathbf{L}$

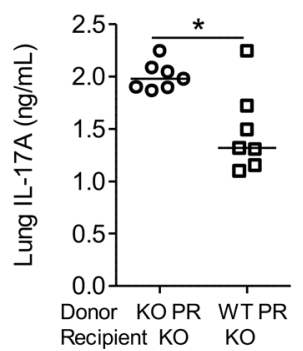

Figure 4. Administration of WT PRs enriched in TSP-1 to Thbs1/mice after Pseudomonas aerugino$s a$ infection reduce lung bacterial burden, airspace neutrophil recruitment, and lung NE activity when compared with Thbs1/- PR-treated mice. (A) Platelet releasates (PRs) from WT mice and Thbs1/- mice were analyzed for TSP-1 isoforms by Western blot using an anti-TSP1 antibody. Purified human TSP-1 was used as positive control. (B-M) Thbs $1^{-1-}$ mice (KO) were inoculated with $P$. aeruginosa strain PA14 at $10^{6}$ inoculum, and mice were subsequently administered either WT PRs or Thbs 1/- PRs by i.p. injection. Outcome measurements (B) lung $\mathrm{CFU} / \mathrm{ml}$, (C) total bronchoalveolar lavage (BAL) cells counts/ml, (D) total BAL PMN counts/ml, (E) lung myeloperoxidase (MPO) activity, and (F) lung neutrophil elastase (NE) activity were obtained 20 hours after infection. (G) G-CSF, (H) GM-CSF, (I) keratinocyte chemoattractant (KC), (J) IL-6, (K) IL-10, (L) IL-17A, and (M) TNF- $\alpha$ concentrations were measured in lung tissue homogenates. Each data point represents an individual mouse, $n$ $=7$ mice per group. Lines indicate the median. ${ }^{*} P<0.05,{ }^{*} P<0.01$ by 2-tailed Mann-Whitney $U$ test.

Administration of dipeptide LasBI after infection reduces lung bacterial burden, and exaggerated neutrophilic inflammation in TSP-1-deficient mice prone to P. aeruginosa intrapulmonary infection. Given that TSP-1 inhibits LasB in vitro, we examined whether disabling pathogen LasB activity corrects the hypersusceptible phenotype of Thbs $1^{-1-}$ mice following infection-induced injury. Following administration of LasBI 2 hours after infection, Thbs $1^{-/-}$mice show reduced lung bacterial burden compared with vehicle-treated Thbs $1^{-1-}$ mice or vehicletreated WT mice (Figure 7A). LasBI administration also reduces airspace neutrophil counts after infection in Thbs $1^{1^{--}}$mice compared with vehicle-treated Thbs $1^{-/-}$mice (Supplemental Figure 3). Moreover, LasBI administration curtails lung tissue concentrations of G-CSF, GM-CSF, IL-6, and L-17A, but not KC, IL-10, and TNF- 0 in Thbs $1^{-/-}$mice compared with vehicle administration (Figure 7, B-H). Thus, curtailing the pathogen protease LasB improves host defense and mitigates the inflammatory response in hypersusceptible Thbs $1^{-1-}$ mice.

Disabling P. aeruginosa type II secretion system or pathogen LasB activity reduces lung bacterial burden, and exaggerated inflammatory injury in TSP-1-deficient mice. The type II secretion apparatus regulates the efflux of a 
A

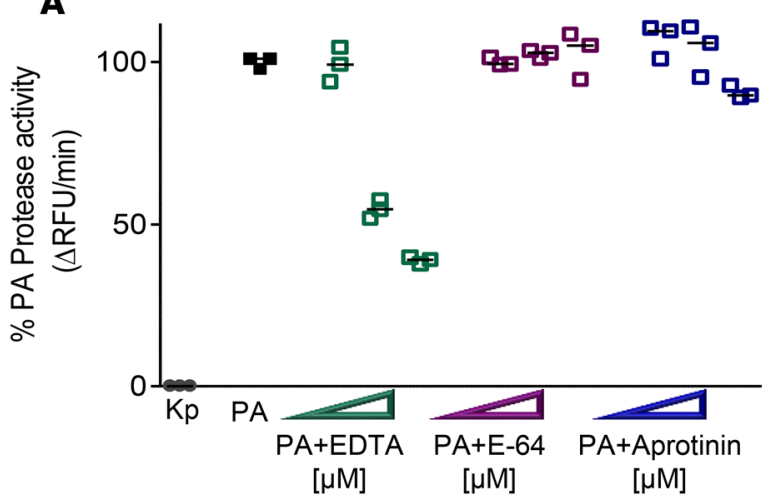

C

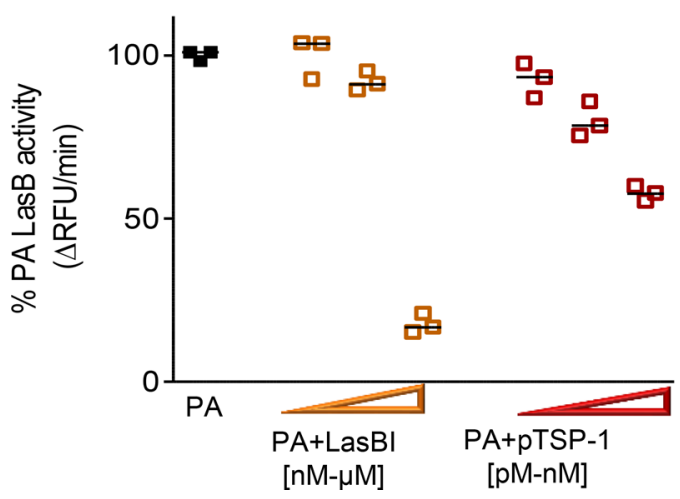

B

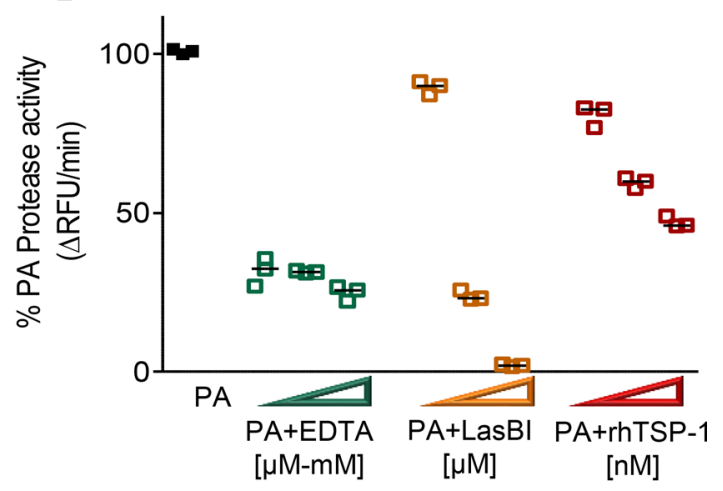

D

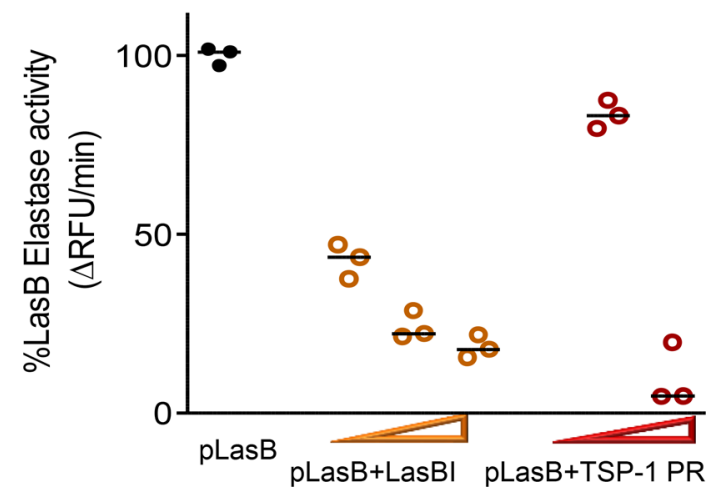

Figure 5. The majority of protease activity secreted by Pseudomonas aeruginosa (PA) exhibits the profile of a metalloprotease and TSP-1 shows dosedependent inhibition of PA exoprotease activity. (A) PA cell-free supernatant (SN) was tested for total protease activity by measuring the hydrolysis of fluorogenic casein substrate over time in the presence or absence of inhibitor. Increasing width of arrowheads indicates increasing molar concentrations of inhibitors. Although SN from Klebsiella pneumoniae (Kp) grown under the same conditions shows no detectable protease activity, that from PA exhibits robust protease activity that is inhibited by EDTA $(20,50$, and $100 \mu \mathrm{M})$. The effects of serine protease inhibitor aprotinin $(20,50$, and $100 \mu \mathrm{M})$, and cysteine protease inhibitor E-64 (20,50, and $100 \mu \mathrm{M})$ on PA protease activity are shown for comparison. (B) Recombinant human TSP-1 (rhTSP-1) dose-dependently inhibits PA protease activity (rhTSP-1 at 78, 156, and $312 \mathrm{nM}$ ). This is compared with the inhibitory activity of EDTA ( $50 \mu \mathrm{M}, 500 \mu \mathrm{M}$, and $5 \mathrm{mM}$ ), and specific LasB inhibitor (LasBI) $N$-mercaptoacetyl-Phe-Tyr-amide (1, 10, and $100 \mu \mathrm{M}$ ). PA SN in the absence of inhibitor is the reference control set as $100 \%$ protease activity. (C) Human purified TSP-1 (200 pM, $1 \mathrm{nM}$, and $78 \mathrm{nM})$ dose-dependently inhibits PA LasB activity as measured by hydrolysis of specific LasB substrate aminobenzoyl-Ala-Gly-Leu-Ala- $p$-nitro-benzyl-amide. This is compared with the inhibitory activity of LasBI (78 nM, $312 \mathrm{nM}$, and $20 \mu \mathrm{M})$. (D) Purified LasB protein (pLasB) was incubated with human TSP-1 from thrombin-activated platelet releasates (PRs) at $1 \mu \mathrm{g}$ or $10 \mu \mathrm{g}$ total protein concentrations and elastase activity measured by hydrolysis of elastin substrate over time. LasBI ( $500 \mathrm{nM}, 50 \mu \mathrm{M}$, and $100 \mu \mathrm{M})$ was incubated with pLasB protein and the degree of percentage pLasB elastase activity inhibition is shown as a comparison. (A-D) Assays were performed in triplicate and a representative study of 3 independent experiments is shown. Lines indicate the median.

number of proteases including LasB (38). XcpQ is an outer membrane protein of the type II secretion system that controls the gating of the secretory channel (38). The in-frame deletion mutant $\triangle x c p Q$ is defective in exoprotease activity when compared with the WT parent strain PA14 (Figure 8A). Inoculation with $\Delta x c p Q$ mutant strain fails to induce the exaggerated lung injury response in hypersusceptible $T h b s 1^{-1-}$ mice when compared with infection induced by the PA14 parent strain (Figure 8, B-G).

The PA14lasB::Tn5 mutant defective in protease, elastase, and LasB activity shows an in vitro growth pattern similar to that of the WT PA14 parent strain (Supplemental Figure 4). Consistent with Thbs $1^{-1-}$ mice infected with the $\triangle x c p Q$ mutant, Thbs $1^{1^{--}}$mice infected with the las $B:: \operatorname{Tn} 5$ mutant show lower bacterial burden and reduced lung inflammatory response compared with mice infected with the WT PA14 parent strain (Supplemental Figure 5). WT mice also show reduced lung bacterial burden and inflammatory injury when administered LasBI after $P$. aeruginosa infection or when infected with the las $B$ mutant compared with respective controls (Supplemental Figure 6). In contrast, infection with the $\Delta p s c D$ mutant strain defective in components of the type III secretion system renders only partial protection to the host in WT mice (Supplemental Figure 7) (39). Collectively, these data indicate that pathogen proteolytic activity, secreted 


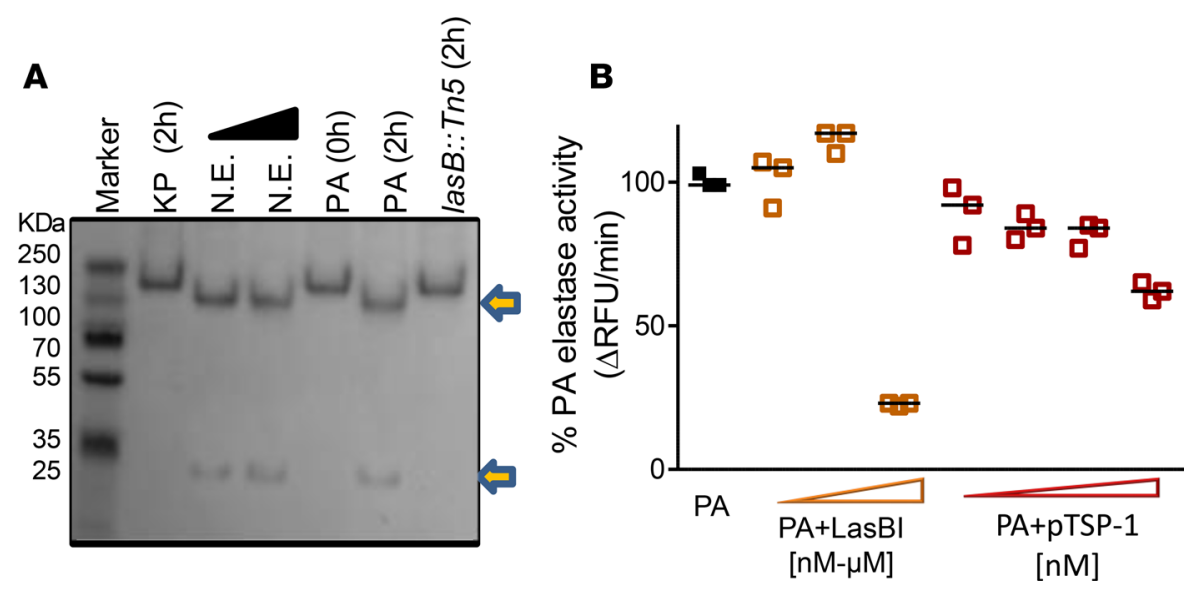

Figure 6. TSP-1 is cleaved by Pseudomonas aeruginosa (PA) into 2 fragments but TSP-1 displays inhibitory activity against PA elastase. (A) Cleavage of TSP-1 by PA cell-free supernatant (SN) was examined by SDS-PAGE, where recombinant human TSP-1 was incubated with Klebsiella pneumoniae (KP) SN, purified neutrophil elastase (NE) (17 and $170 \mathrm{nM}$ ), PA14 SN, or PA14 lasB::Tn5 mutant SN (lacking exoprotease activity) at $37^{\circ} \mathrm{C}$ for 0 and 2 hours. While KP and lasB::Tn5 mutant SN failed to cleave TSP-1, NE and PA14 SN cleaved TSP-1 similarly into 2 fragments represented by $130-k D a$ and $~ 30-k D a$ bands. Arrows indicate cleaved products. (B) Although TSP-1 is cleaved by PA14, TSP-1 inhibits PA14 elastase activity. The effect of increasing molar concentrations of purified human TSP-1 (78, 156, 312, and $624 \mathrm{nM})$, and LasBI (624 nM, $10 \mu \mathrm{M}$, and $100 \mu \mathrm{M})$ on PA14 elastase activity are shown. Increasing molar concentrations of inhibitor are indicated by increasing width of arrowheads. The elastase activity was measured for 2 hours after the substrate was added to the reaction. The SDS-PAGE experiment was performed twice. Data points for elastin cleavage assay indicate technical replicates and the experiment is representative of 3 independent studies. Lines indicate the median. RFU, relative fluorescence units.

by the type II secretion system, is a major instigator of unbridled inflammatory injury and that host TSP-1 counters this response.

TSP-1 inhibits NE activity from neutrophils isolated from the airways of mechanically ventilated ICU patients. Overactive neutrophilic inflammation in $T h b s 1^{-{ }^{-}}$mice may be the consequence of ineffective host curtailment of pathogen burden due to unopposed LasB virulence factor. However, TSP-1 may also provide an additional line of defense against tissue injury by directly subduing neutrophil-derived proteolysis, as

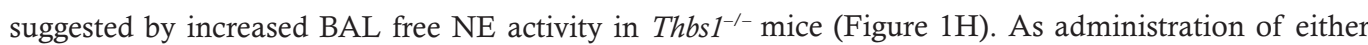
TSP-1 or PRs enriched in TSP-1 dampen lung NE activity in vivo (Figure 3E and Figure 4F), we further show that TSP-1 from both mouse and human PRs can directly inhibit purified NE activity and rhTSP-1 dose-dependently inhibits NE enzymatic function in vitro (Figure 9, A and B). We isolated neutrophils from airway secretions of mechanically ventilated ICU patients using a closed-loop inertial microfluidic sorter technique to enhance separation resolution of specific leukocyte subsets from viscous biofluids (40). This method recovers viable cells, the vast majority of which are PMNs (Figure 9, C and D). We previously showed that airway-derived human neutrophils exhibit a primed activated state compared with blood-derived neutrophils, presumably due to factors that initially trigger the migration of these cells from the bloodstream to the airways (40). Indeed, neutrophils recovered from the lower respiratory tract of mechanically ventilated ICU patients exhibit a primed activated state, as suggested by the inability of phorbol 12-myristate 13-acetate (PMA) to further enhance NE release (Figure 9E). Moreover, human PRs enriched in TSP-1 dose-dependently inhibit NE activity from airway neutrophils recovered from patients. Collectively, these findings suggest that TSP-1 released from activated platelets may provide an additional line of defense by curbing tissue injury through inhibition of neutrophil proteolytic activity.

\section{Discussion}

The main finding of this study is that TSP-1 provides a dual mechanism of host protection against pathogeninitiated and host-sustained extracellular matrix proteolysis. The data support the contention that TSP-1 disarms a pathogen-encoded protease exploited by $P$. aeruginosa. TSP-1 improves host resistance to the virulence of a pathogen through direct inhibition of a pathogen-encoded metalloprotease and enhances host resilience by curbing continued tissue injury through inhibition of neutrophil proteolytic activity. $P$. aeruginosa possesses an extensive armamentarium of virulence factors to counter host defenses including the secretion of proteases. In particular, the metalloendopeptidase LasB degrades extracellular matrix constituents such as collagen and elastin to induce tissue injury (11). Our findings show that TSP-1 dose-dependently 
A

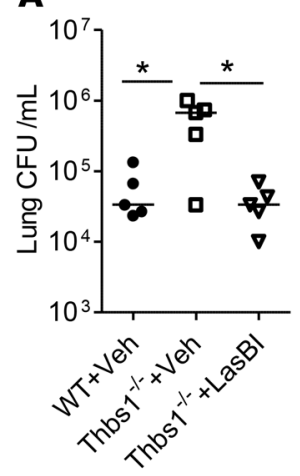

E

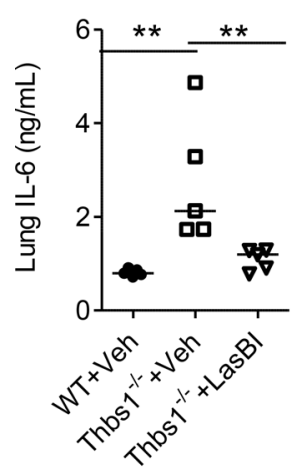

B

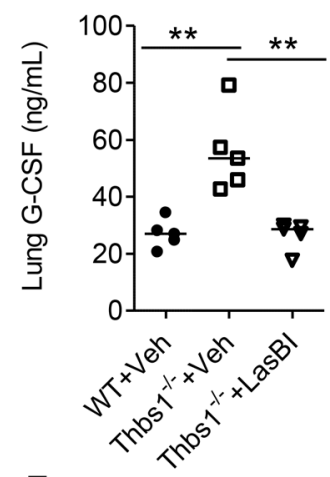

$\mathbf{F}$

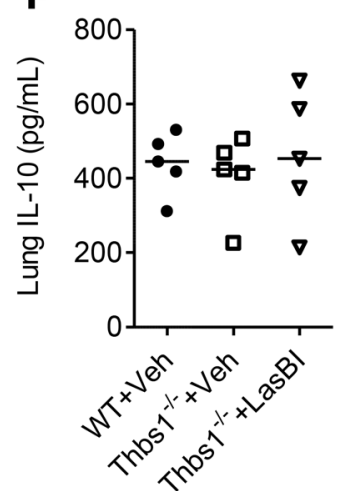

C

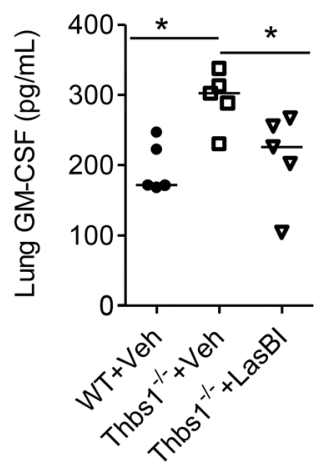

G

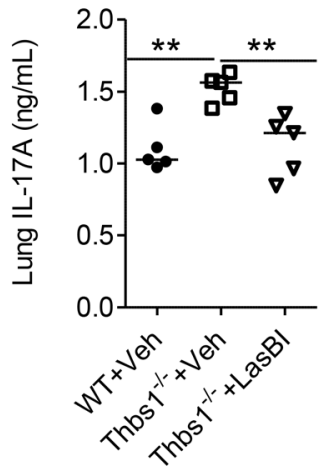

D

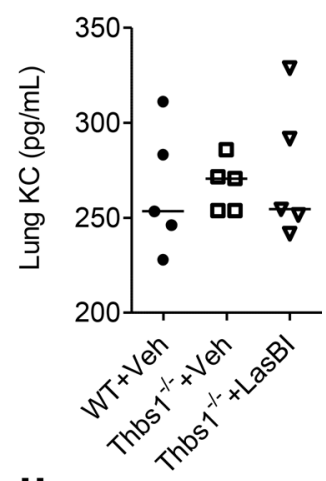

H

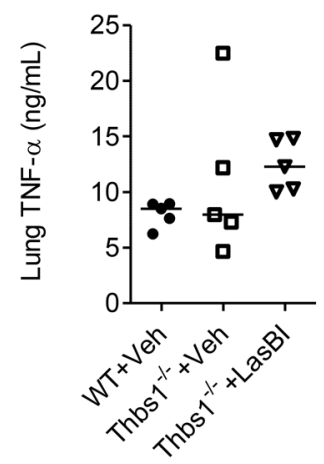

Figure 7. Administration of dipeptide compound LasB inhibitor after infection reduces lung bacterial burden and inflammatory cytokines in

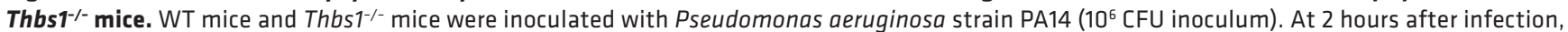
mice were administered either vehicle (DMSO) or LasB inhibitor (LasBI) at $200 \mu \mathrm{g} /$ mouse by i.p. injection. Outcome measurements were obtained 20 hours after infection. (A) Lung CFU/ml, (B) G-CSF, (C) GM-CSF, (D) keratinocyte chemoattractant (KC), (E) IL-6, (F) IL-10, (C) IL-17A, and (H) TNF- $\alpha$ concentrations were measured in lung tissue homogenates. Each data point represents an individual mouse, $n=5$ mice per group. Lines indicate the median. ${ }^{*} P<0.05,{ }^{*} P<0.01$ by Kruskal-Wallis test with Dunn's multiple comparisons.

curtails PA14 exoprotease activity, and LasB enzymatic function in vitro. Incubation with PA14 cell-free supernatant results in proteolytic cleavage of TSP-1 into 2 fragments, a smaller 30-kDa and a larger 130-kDa fragment. Despite cleavage of TSP-1 by high levels of pathogen-derived exoproducts in cell-free supernatant, TSP-1 displays potent inhibitory activity against Pseudomonas elastolytic activity in vitro, similar to the findings of TSP-1 cleavage by NE (37). In support of the in vitro findings, Thbs $1^{-/-}$mice show reduced survival, failure to regain weight, impaired host defense, and increased susceptibility to $P$. aeruginosa-induced lung inflammatory injury in vivo. Reconstitution of TSP-1 corrects the impaired host defense in Thbs $1^{-1-}$ mice, and the accompanying aberrant host injury response initiated by $P$. aeruginosa challenge, suggesting that the inability to mount an effective host protection program due to a TSP-1-deficient state results in amplified neutrophilic inflammation.

When LasBI is administered after infection, the hypersusceptible phenotype of Thbs $1^{-1-}$ mice is corrected with reductions in lung bacterial burden, airspace neutrophil counts, and lung tissue inflammatory cytokines. Consistent with LasBI administration, mice infected with PA14 mutant strains characterized by disabled proteolytic activity show reduced lung bacterial burden and neutrophilic inflammation compared with mice infected with PA14 parent strain. Collectively, these data indicate that LasB is the predominant protease secreted by PA14 and a main instigator of unbridled neutrophilic inflammation in mice deficient in TSP-1. TSP-1 not only curtails LasB proteolytic activity but also provides an additional line of protection by directly tempering NE enzymatic function following downstream neutrophil activation. Thus, TSP-1 provides a dual strategy to disarm a pathogen-secreted protease and secondary trigger of aberrant hostsustained extracellular matrix proteolysis.

TSP-1 inhibits serine proteases plasmin, cathepsin G, and NE in vitro $(36,37,41,42)$. The TSR3 domain of TSP-1 contains sequences bearing striking similarity to the Kazal family of Streptomyces subtilisin serine protease inhibitors $(41,43)$. We previously showed that peptides corresponding to discrete 
A

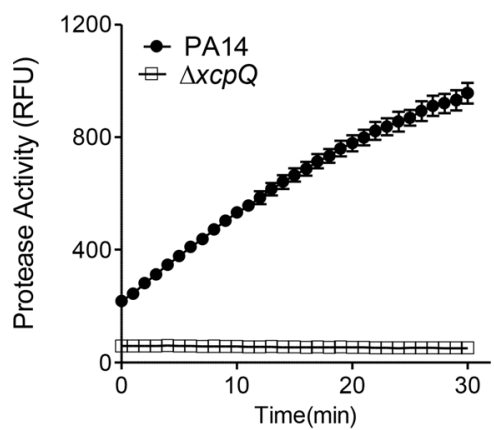

B

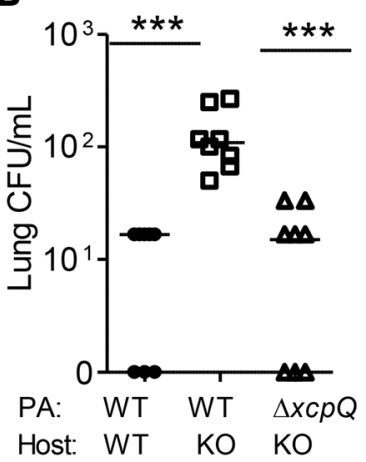

$c$

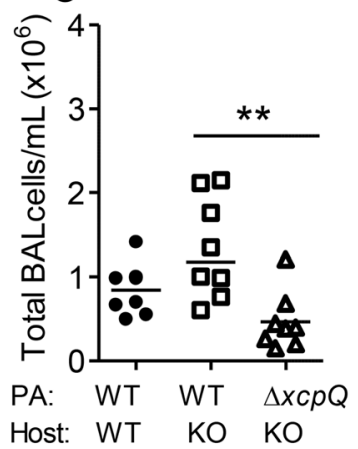

D

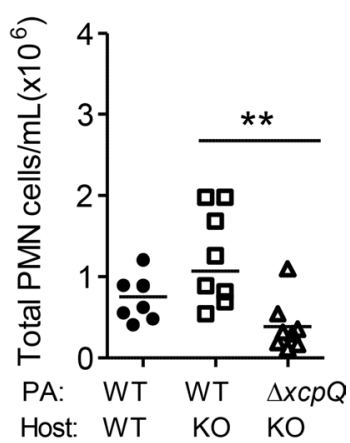

Figure 8. The $\triangle x \subset p Q \underline{Q}$ mutant induces less bacterial burden, airspace neutrophil recruitment, and inflammation in Thbs 1/- $^{-1}$ mice when compared with Pseudomonas aeruginosa parent strain. WT and Thbs $1^{-1-}$ (KO) mice were inoculated with $P$. aeruginosa (PA) parent strain PA14 or $\triangle x c p \underline{Q}$ mutant strain and outcome measurements obtained 20 hours after infection (PA inoculum, $2.2 \times$ $10^{6} \mathrm{CFU} ; \Delta x c p Q$ mutant inoculum, $2.5 \times 10^{6}$ CFU). (A) Total protease activity of cell-free supernatant (SN) from the PA14 parent strain and $\triangle x c p Q$ mutant was measured by the cleavage of fluorogenic casein substrate in relative fluorescence units (RFU) over time. (B) Lung CFU/ml, (C) total bronchoalveolar lavage (BAL) cell counts/ml, (D) total BAL PMN counts/ml, (E) total BAL protein concentration, (F) BAL free neutrophil elastase (NE) activity, and (G) lung tissue myeloperoxidase (MPO) activity were measured in WT and KO mice. Each data point represents an individual mouse, $n=8$ mice/group. Lines indicate the median. ${ }^{*} P<0.05,{ }^{* *} P<0.01$, ${ }^{* * *} P<0.001$ by Kruskal-Wallis test with Dunn's multiple comparisons.

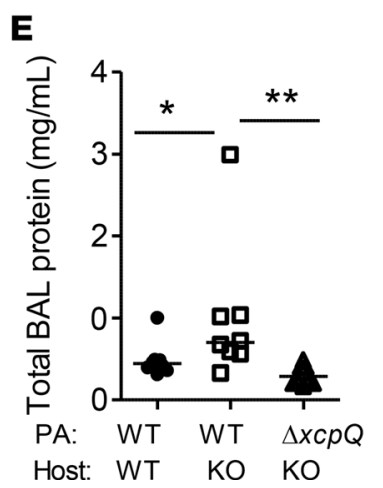

Host: WT KO KO

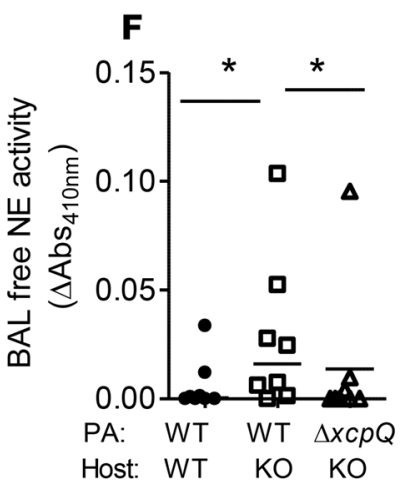

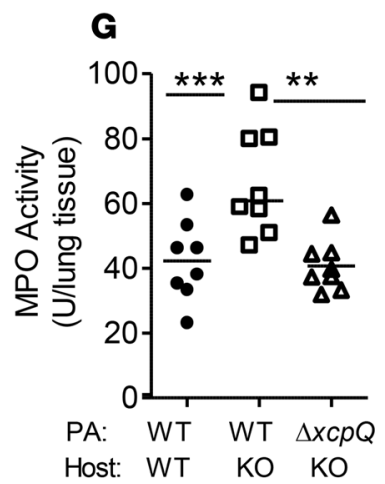

regions within the TSR3 domain can restrain neutrophil-derived elastase and cathepsin G enzymatic function in vitro (28). Moreover, TSP-1 bridles the proteolytic action of neutrophils leading to impaired host defense in a $K$. pneumoniae infection model where unopposed serine protease activity appears beneficial for early bacterial containment and elimination by phagocytes (28). In contrast, our in vitro and in vivo findings in the acute $P$. aeruginosa infection model support the notion that pathogen-encoded proteases such as LasB trigger a robust lung injury phenotype that diverts host defense mechanisms and escalates neutrophil tissue damage response. Mice deficient in TSP-1 are hypersusceptible to the injurious effects and attest to the fact that failure to effectively counter tissue-damaging proteolysis during infection can result in dysregulated neutrophilic inflammation.

Originally described as an inhibitor of angiogenesis (44), TSP-1 is a homotrimeric glycoprotein possessing the following distinct structural modules (in sequence from the $\mathrm{NH}_{2}$ terminal to $\mathrm{COOH}$ terminal globular domains): a heparin binding region, a procollagen-like domain harboring a cysteine-rich region homologous to the $\mathrm{NH}_{2}$ terminal pro-peptide of type I procollagen $\alpha 1$-chain (45), properdin-like type I repeats (TSR1), epidermal growth factor-like type II repeats (TSR2), and a $\mathrm{Ca}^{2+}$-binding TSR3 region $(46,47)$. The TSP family of proteins comprise at least 5 proteins (TSP1-5) that share similar TSR2 and TSR3 regions $(16,48)$, with TSP-1 and TSP-2 exhibiting additional similarities including the procollagen-like homology region and 3 properdin-like TSR1 domains. TSP-1 has been shown to inhibit matrix metalloproteinase 3-dependent (MMP-3-dependent) activation of MMP-2 and MMP-9 zymogens in vitro (47). 
A

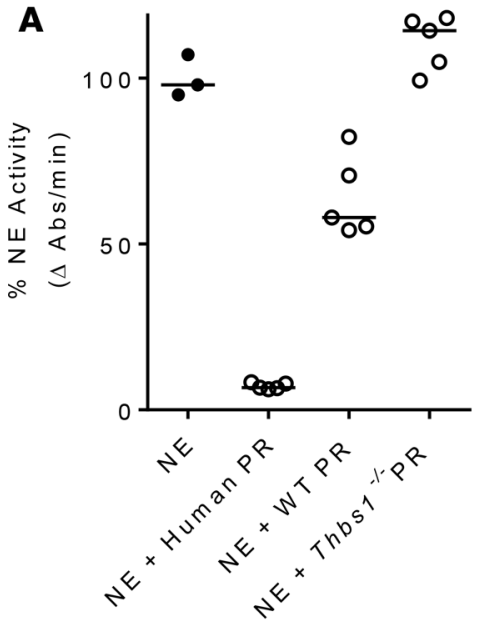

D

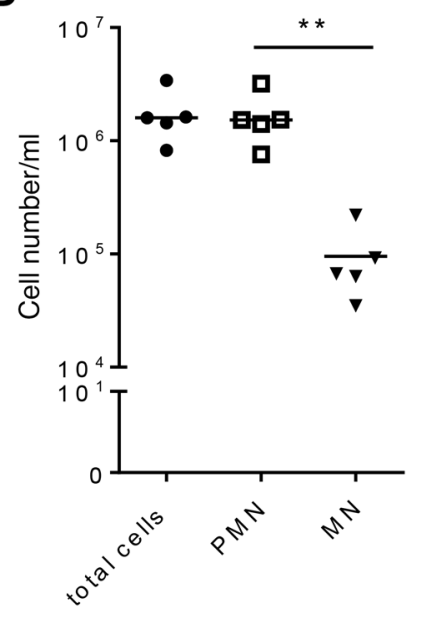

B

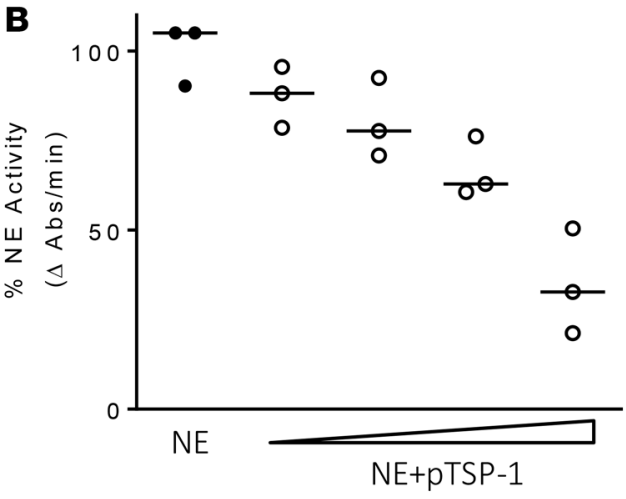

C

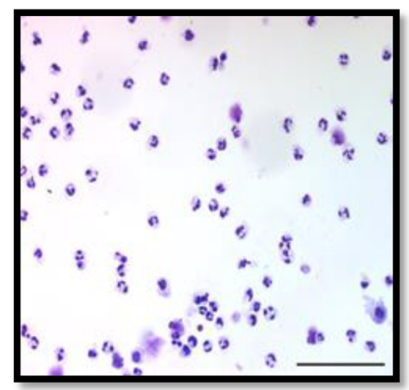

$\mathbf{E}$

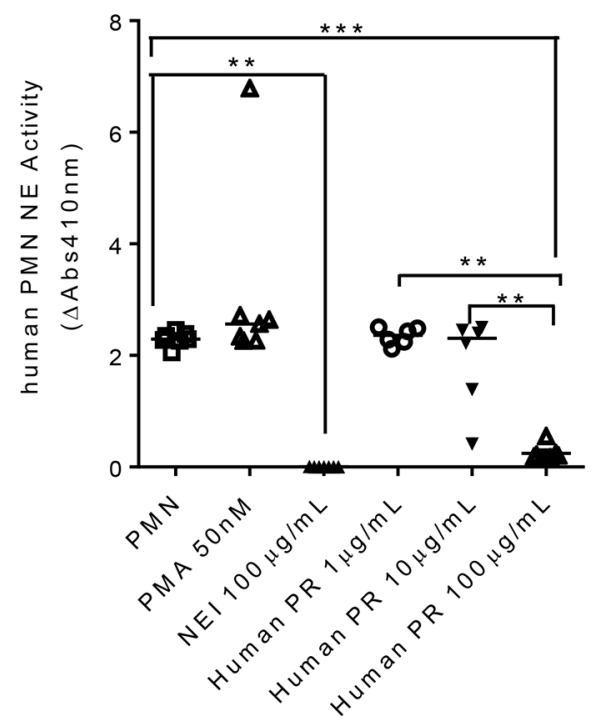

Figure 9. Platelet releasates enriched in TSP-1 dose-dependently inhibit NE activity from neutrophils isolated from tracheal secretions of mechanically ventilated ICU patients. (A) Neutrophil elastase (NE) was incubated with human platelet releasates (PRs), WT mouse PRs, or Thbs $7^{-1-}$ mouse PRs for 24 hours and NE activity measured as the rate of enzymatic hydrolysis of NE substrate $N$-methoxysuccinyl-Ala-Ala-Pro-Val $p$-nitroanilide reflected by the increase in absorbance (Abs) at $410 \mathrm{~nm}$ over time. Data points indicate combined data from 2 independent studies using PRs pooled from 6-8 mice/ group. Lines indicate the median. (B) Human NE was incubated with human TSP-1 at different concentrations (38.8, 77.6, 194, and 388 nM) for 10 hours and NE activity measured as the rate of enzymatic hydrolysis of NE substrate $N$-methoxysuccinyl-Ala-Ala-Pro-Val $p$-nitroanilide reflected by the increase in Abs at $410 \mathrm{~nm}$ over time. Data points indicate technical replicates from 1 experiment. Lines indicate the median. (C) Leukocytes were collected from human airway secretions, and cytospin shows predominance of neutrophils. Scale bar: $100 \mu \mathrm{m}$. (D) Cell count and differential of isolated leukocytes. PMN, polymorphonuclear cells; MN, mononuclear cells. Each data point was obtained from individual patient tracheal samples $(N=5)$. (E) Isolated human airway neutrophils were incubated with PMA (50 nM), NE inhibitor $(100 \mu \mathrm{g} / \mathrm{ml}, \mathrm{NEI})$, or human PR $(1,10$, and $100 \mu \mathrm{g} / \mathrm{ml})$. Each data point was obtained from individual patient tracheal samples $(N=7)$. Lines indicate the median. ${ }^{* *} P<0.01,{ }^{* * *} P<0.001$ by ANOVA with Tukey's multiple comparisons test.

Despite the structural similarity of the gelatinases MMP-2 and MMP-9, TSP-1 inhibits MMP-3 activation/ processing of pro-MMP-9 but not pro-MMP-2 in an in vivo tumor progression model, highlighting the need for in vivo confirmation to better understand the physiological contexts that bring to bear the biological role of TSP-1, given numerous binding partners in vitro (49). The ability of TSP-1 to curtail LasB, a secreted virulence factor, and regulate proteolytic injury during acute bacterial lower respiratory tract infection provide a new dimension to host-pathogen interplay and the biological function of TSP-1 in providing a check on exuberant host inflammatory responses following microbial invasion.

TSP-1 is predominantly expressed in the vasculature by platelet $\alpha$-granules (20), locally by the lung endothelium $(24,50)$, and released into the extracellular milieu following injury and inflammation. Platelet-derived 
proteins including TSP-1 are found in the airspaces of ARDS patients and correlate with composite injury scores (23), but how they facilitate the course of inflammation and injury has been poorly understood. Certain microbes such as $P$. aeruginosa can trigger a cascade of injury and cell death through the release of exoproducts such as LPS, flagellin, exotoxins, lipases, and proteases, produce breakage of tight junctional gaps, degrade extracellular matrix proteins that constitute the basement membrane, and potentially cause alveolar barrier disruption that is the hallmark of lung injury (51-57). The host protects against aggressive lung injury triggered by infection through the release of soluble factors from injured blood vessels and platelets. When this protective mechanism goes awry, such as in the case of endothelial cell death following severe injury or when platelet counts fall below a threshold level during overwhelming critical illness (58-60), the host may be unable to adequately repair the alveolar barrier required for adequate gas exchange. Our findings support the notion that TSP-1 promotes the course of repair following lung injury by curtailing pathogen-initiated proteolysis and subduing inflammation.

\section{Methods}

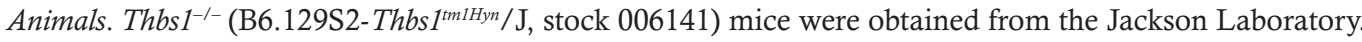
Thbs $1^{-1-}$ mice were backcrossed to C57BL/6J mice for 9 generations prior to sending to the Jackson Laboratory. Thbs $1^{-1-}$ mice were subsequently backcrossed an additional 4 generations to C57BL/6J mice at the University of Pittsburgh facilities. All experimental procedures were performed in age-matched (8-12 weeks old) and sex-matched mice in randomized fashion. All mice were maintained in a specific pathogen-free environment, housed in the same vivarium, and fed the same standard chow for at least 4 weeks prior to the commencement of experiments. A technician who was blinded to the experimental hypothesis conducted the experiments and, depending upon mouse availability, experiments were conducted with precalculated sample size either as a single experiment or divided into 2 independent experiments.

Bacterial strains and genetic engineering. To verify that the PA14lasB::Tn5 nonredundant transposon insertion mutant accumulated no secondary mutations that could influence bacterial phenotypes studied here, the complete genome sequences of the WT and mutant strains were sequenced on an Illumina NextSeq500 and their genomes compared using breseq 0.30 (61). The PA14lasB::Tn5 mutant had a mini-Tn5 insertion in the expected location (62) as well as a deletion of a selfish genetic island comprising 25 genes (PA14_15350, PA14_15600). The in-frame deletion mutant PA14 $\Delta p s c D$ has been previously reported (39). The in-frame deletion PA14 $\triangle x c p Q$ was made using allelic replacement with pMQ30 as previously described (63) and in vivo experiments conducted with this mutant utilized its own reference parent PA14 strain.

Experimental bacterial pneumonia model. P. aeruginosa PA14 is a reference clinical strain originally isolated from a burn patient (32). A standard protocol was used to grow PA14. A mutant PA14 containing a transposon insertion in the lasB gene (PA14lasB::Tn5, abbreviated as lasB) and in-frame deletion mutants PA14 $\triangle x c p Q$ and PA14 $\triangle p s c D$ were utilized in select experiments. Briefly, a frozen stock of bacteria was grown in Luria-Bertani (LB) broth for 18 hours at $37^{\circ} \mathrm{C}$. The concentration of bacteria was estimated by measuring the $\mathrm{OD}_{600 \mathrm{~nm}}$ and confirmed by quantifying colony-forming units (CFU) of the inoculum instilled into mice for each experiment. To prepare the inoculum, bacteria were pelleted by centrifugation at $1,864 \mathrm{~g}$ for 15 minutes, washed twice, and resuspended in sterile PBS before use. WT and Thbs $1^{-1-}$ mice were anesthetized with isoflurane, and $100 \mu \mathrm{l}\left(10^{6}\right.$ CFU) of bacterial slurry was carefully administered i.t. under direct visualization using a sterile 200- $\mu 1$ pipettor with the filtered tip positioned just above the vocal cords. The synthetic dipeptide LasBI $N$-mercaptoacetylPhe-Tyr-amide previously shown to inhibit $P$. aeruginosa biofilm production in vitro with a $\mathrm{K}_{\mathrm{i}}$ of $41 \mathrm{nM}$ was generated (13) (CHI Scientific, Inc) and administered intraperitoneally (i.p.) 2 hours after infection. For determining inocula concentrations, the $P$. aeruginosa slurry was serially diluted, and CFU were counted on LB agar plates following an overnight incubation at $37^{\circ} \mathrm{C}$. In select experiments, purified TSP-1 isolated from human PRs (Athens Research and Technology) at $125 \mu \mathrm{g} /$ mouse, or PRs from donor WT or Thbs $1^{-/-}$mice at $130 \mu \mathrm{g} /$ mouse were administered i.p. to recipient mice following acute intrapulmonary infection with PA14.

Mouse necropsies, BAL, and measurement of bacterial burden. Mice were euthanized 20 hours after $P$. aeruginosa inoculation with overdose of isoflurane administration, followed by cardiac puncture and exsanguination. Mouse necropsy, lung tissue processing, and BAL harvesting were performed as previously described $(28,64)$. The left lung and spleen were removed following euthanasia. For enumerating bacterial CFU in the lungs and spleens, tissues were homogenized in $1 \mathrm{ml}$ of sterile deionized water. Tissue homogenates were plated by 10 -fold serial dilution on LB agar plates. BAL total protein concentration was determined by using the Pierce BCA Protein Assay Kit (Thermo Fisher Scientific). 
SDS-PAGE and Western blot. A precast NuPAGE 4\%-12\% or 12\% Bis-Tris Mini Gel, 1.5 mm, 10 well (Life Technologies) was used to perform SDS-PAGE. A constant voltage (120 V) was applied to perform electrophoresis. Gels were stained with Coomassie Blue R250 in 50\% methanol, 10\% acetic acid, and 40\% $\mathrm{H}_{2} \mathrm{O}$ for 5 minutes followed by a wash step with Milli-Q water.

MPO assay. Method for measurement of MPO activity in lung tissues has been previously described (28). Hexadecyl-trimethylammonium bromide $(0.5 \%)$ buffer was added to homogenized lung tissue. Following sonication on ice, samples were centrifuged at $20,000 \mathrm{~g}$ for 4 minutes at $4^{\circ} \mathrm{C}$. The supernatant was transferred to a 96-well plate and change in absorbance over time was measured following addition of $o$-dianisidine dihydrochloride solution. For calculation of the MPO lung tissue content, the following formula was used: ( $\triangle$ Absorbance [between 0 and $60 \mathrm{~s}] /$ time $[\mathrm{min}] \times 1) /\left(1.13 \times 10^{-2}\right)$.

Protease, elastase, LasB elastase, and LasB activity assay. P. aeruginosa isolates were grown in LB broth overnight and their cell-free supernatants were tested for total exoprotease and elastase activity using fluorogenic casein and elastin substrates, respectively. The samples were measured with a filter fluorimeter at excitation $485 \mathrm{~nm}$ and emission $528 \mathrm{~nm}$ (SpectraMax i3X, Molecular Devices). An EnzChek Protease Assay Kit and EnzChek Elastase Assay Kit were used for measuring protease and elastase activity (Thermo Fisher Scientific). The enzymatic hydrolysis of fluorogenic casein, elastin, or LasB substrate (aminobenzoyl-Ala-Gly-Leu-Ala-p-nitro-benzyl-amide) over time was measured in cell-free bacterial supernatant in the absence or presence of inhibitors aprotinin, E-64, EDTA (Sigma-Aldrich), or specific LasBI. In select experiments, the inhibitory action of purified TSP-1 isolated from PRs (Athens Research and Technology), rhTSP-1 (R\&D Systems), or TSP-1 enriched in PRs was examined.

NE activity measurements. NE activity in supernatant of the lung tissue homogenates and cell-free BAL fluid were measured utilizing a previously described method $(28,65)$. Briefly, $50 \mu 1$ of BAL or diluted supernatant of lung homogenates was incubated with $0.1 \mathrm{M}$ Tris- $\mathrm{HCl}$ buffer $(\mathrm{pH}$ 8.0) containing 0.5 $\mathrm{M} \mathrm{NaCl}$ and $0.24 \mathrm{mM} \mathrm{NE}$ substrate $N$-methoxysuccinyl-Ala-Ala-Pro-Val $p$-nitroanilide (Sigma-Aldrich) at $37^{\circ} \mathrm{C}$ for 24 hours. The degradation of substrate in samples was measured by spectrophotometry at $410 \mathrm{~nm}$ over time.

Measurement of cytokines and total protein. Cytokines were measured in samples of lung tissue homogenates using Duoset antibodies to perform enzyme-linked immunosorbent assay (R\&D Systems). Levels of cytokines and chemokines were measured from left lung tissue homogenates in PBS buffer. Total protein concentrations were measured using the Pierce BCA Protein Assay Kit.

Isolation of PRs. Preparation of mouse and human PRs enriched in TSP-1 was a modification of a previously published report (24). Briefly, mouse or human blood was collected in tubes containing the anticoagulant citrate phosphate dextrose (CPD) (66) and blood was centrifuged at $100 \mathrm{~g}$ for 10 minutes at room temperature to enrich for plasma. Plasma was centrifuged at room temperature for an additional 10 minutes at $100 \mathrm{~g}$ and contaminating red and white blood cells were removed. The platelet suspension was subsequently transferred to a new tube and centrifuged at $1,000 \mathrm{~g}$ for 10 minutes. The concentrated platelet pellets were then resuspended in Tyrode's buffer and incubated for 10 minutes at $37^{\circ} \mathrm{C}$ prior to stimulation with $1 \mathrm{U} / \mathrm{ml}$ thrombin for 20 minutes at $37^{\circ} \mathrm{C}$. Cells were centrifuged at $1,000 \mathrm{~g}$ for 10 minutes and the supernatant was collected. PRs were examined by immunoblot to assess for TSP-1 using a specific antiTSP-1 antibody (clone A6.1, Thermo Fisher Scientific).

Neutrophil isolation from airways of mechanically ventilated patients. Deidentified tracheal aspirates were obtained from adult mechanically ventilated patients using a modification of a previously published protocol (67). When clinically indicated, airway secretions were obtained using a 14F/channel, 4.67-mm diameter suction catheter. A $10 \mathrm{ml}$ prefilled syringe containing sterile $0.9 \%$ normal saline was attached to the end of the suction ballard and $5 \mathrm{ml}$ of $0.9 \%$ normal saline was delivered directly into the airways. Secretions were suctioned when the ballard was fully advanced and collected into a sterile sputum collection cup. The sputum container was placed on ice and immediately processed by mechanical disruption in PBS by gentle pipetting. We have recently reported the methods for isolation of neutrophils from airway secretions using a closed-loop inertial spiral microfluidics technique (40).

Statistics. For in vitro biochemical assays, the experimental results are reported as the mean plus or minus standard error of the mean unless otherwise indicated. For survival analysis, a log-rank Mantel-Cox test was performed. Percentage weight loss over time was examined by a 2-way ANOVA with Sidak multiple comparisons test. For data analysis obtained from animal experiments, individual data points are represented and the median reported using a 2-tailed Mann-Whitney $U$ test when comparing 
2 groups, and a Kruskal-Wallis test with Dunn's multiple comparisons when comparing more than 2 groups. A $P$ value of less than 0.05 was considered significant. All statistical analyses were performed using GraphPad Prism version 6.0.

Study approval. The Institutional Animal Care and Use Committee at the University of Pittsburgh approved the animal protocol. The IRB approved this exempt study (PRO16060443) to collect airway secretions that are normally discarded during routine clinical care in a de-identified manner from mechanically ventilated patients in the medical ICU of the University of Pittsburgh Medical Center.

\section{Author contributions}

YQ, TO, and RB performed the experiments, analyzed the data, and drafted the manuscript. WB, JZ, MH, ZX, HR, and JT performed the experiments and analyzed the data. JMB, ZC, VSC, RMQS, and MEZ provided critical reagents, interpreted the data, and revised the work for important intellectual content. AR, $\mathrm{PR}, \mathrm{JH}$, and JP interpreted the data and revised the work for important intellectual content. JSL conceived, designed, analyzed, and interpreted the data, and wrote the manuscript.

\section{Acknowledgments}

This work was supported by the NIH/National Heart, Lung, and Blood Institute under award numbers R01HL136143 (to J.S. Lee), R01HL086884 (to J.S. Lee), and P01 HL114453 (to P. Ray), and by the NIH/ National Institute of Allergy and Infectious Diseases under award number R21AI119042 (to J.S. Lee), Cystic Fibrosis Foundation Research Development Program (to J. Pilewski and J.S. Lee), the Vascular Medicine Institute, the Hemophilia Center of Western Pennsylvania, and the Institute for Transfusion Medicine (to J.S. Lee). The content is solely the responsibility of the authors and does not necessarily represent the official views of the NIH.

Address correspondence to: Janet S. Lee, 3459 Fifth Avenue, Montefiore University Hospital NW628, Pittsburgh, Pennsylvania, 15213, USA. Phone: 412.692.2328; Email: leejs3@upmc.edu.

1. Forel JM, et al. Ventilator-associated pneumonia and ICU mortality in severe ARDS patients ventilated according to a lungprotective strategy. Crit Care. 2012;16(2):R65.

2. Iregui MG, Kollef MH. Ventilator-associated pneumonia complicating the acute respiratory distress syndrome. Semin Respir Crit Care Med. 2001;22(3):317-326

3. Bauer TT, Ewig S, Rodloff AC, Müller EE. Acute respiratory distress syndrome and pneumonia: a comprehensive review of clinical data. Clin Infect Dis. 2006;43(6):748-756.

4. Safdar N, Dezfulian C, Collard HR, Saint S. Clinical and economic consequences of ventilator-associated pneumonia: a systematic review. Crit Care Med. 2005;33(10):2184-2193.

5. Timsit JF, Zahar JR, Chevret S. Attributable mortality of ventilator-associated pneumonia. Curr Opin Crit Care. 2011;17(5):464-471.

6. Markowicz P, et al. Multicenter prospective study of ventilator-associated pneumonia during acute respiratory distress syndrome. Incidence, prognosis, and risk factors. ARDS Study Group. Am J Respir Crit Care Med. 2000;161(6):1942-1948.

7. Hakki M, Limaye AP, Kim HW, Kirby KA, Corey L, Boeckh M. Invasive Pseudomonas aeruginosa infections: high rate of recurrence and mortality after hematopoietic cell transplantation. Bone Marrow Transplant. 2007;39(11):687-693.

8. Venier AG, et al. Identifying new risk factors for Pseudomonas aeruginosa pneumonia in intensive care units: experience of the French national surveillance, REA-RAISIN. $J$ Hosp Infect. 2011;79(1):44-48.

9. Carratalà J, Rosón B, Fernández-Sevilla A, Alcaide F, Gudiol F. Bacteremic pneumonia in neutropenic patients with cancer: causes, empirical antibiotic therapy, and outcome. Arch Intern Med. 1998;158(8):868-872.

10. Vincent JL, et al. International study of the prevalence and outcomes of infection in intensive care units. JAMA. 2009;302(21):2323-2329.

11. Strateva T, Mitov I. Contribution of an arsenal of virulence factors to pathogenesis of Pseudomonas aeruginosa infections. Ann Microbiol. 2011;61(4):717-732.

12. Sawa T. The molecular mechanism of acute lung injury caused by Pseudomonas aeruginosa: from bacterial pathogenesis to host response. J Intensive Care. 2014;2(1):10.

13. Cathcart GR, et al. Novel inhibitors of the Pseudomonas aeruginosa virulence factor LasB: a potential therapeutic approach for the attenuation of virulence mechanisms in pseudomonal infection. Antimicrob Agents Chemother. 2011;55(6):2670-2678.

14. Alcorn JF, Wright JR. Degradation of pulmonary surfactant protein D by Pseudomonas aeruginosa elastase abrogates innate immune function. J Biol Chem. 2004;279(29):30871-30879.

15. Le Berre R, et al. Relative contribution of three main virulence factors in Pseudomonas aeruginosa pneumonia. Crit Care Med. 2011;39(9):2113-2120.

16. Bornstein P. Diversity of function is inherent in matricellular proteins: an appraisal of thrombospondin 1. J Cell Biol. 1995;130(3):503-506. 
17. Iruela-Arispe ML, Bornstein P, Sage H. Thrombospondin exerts an antiangiogenic effect on cord formation by endothelial cells in vitro. Proc Natl Acad Sci USA. 1991;88(11):5026-5030.

18. Sweetwyne MT, Murphy-Ullrich JE. Thrombospondin1 in tissue repair and fibrosis: TGF- $\beta$-dependent and independent mechanisms. Matrix Biol. 2012;31(3):178-186.

19. Lawler J. The structural and functional properties of thrombospondin. Blood. 1986;67(5):1197-1209.

20. Lawler PR, Lawler J. Molecular basis for the regulation of angiogenesis by thrombospondin-1 and -2. Cold Spring Harb Perspect Med. 2012;2(5):a006627.

21. Resovi A, Pinessi D, Chiorino G, Taraboletti G. Current understanding of the thrombospondin-1 interactome. Matrix Biol. 2014;37:83-91.

22. Lawler J, Cohen AM, Chao FC, Moriarty DJ. Thrombospondin in essential thrombocythemia. Blood. 1986;67(2):555-558

23. Idell S, et al. Platelet-specific alpha-granule proteins and thrombospondin in bronchoalveolar lavage in the adult respiratory distress syndrome. Chest. 1989;96(5):1125-1132.

24. Lee JH, et al. Lung stem cell differentiation in mice directed by endothelial cells via a BMP4-NFATc1-thrombospondin-1 axis Cell. 2014;156(3):440-455

25. Kreis C, La Fleur M, Ménard C, Paquin R, Beaulieu AD. Thrombospondin and fibronectin are synthesized by neutrophils in human inflammatory joint disease and in a rabbit model of in vivo neutrophil activation. J Immunol. 1989;143(6):1961-1968.

26. Catena $\mathrm{R}$, et al. Bone marrow-derived $\mathrm{Gr}^{+}$cells can generate a metastasis-resistant microenvironment via induced secretion of thrombospondin-1. Cancer Discov. 2013;3(5):578-589.

27. Zhao Y, et al. Thrombospondin-1 triggers macrophage IL-10 production and promotes resolution of experimental lung injury. Mucosal Immunol. 2014;7(2):440-448.

28. Zhao Y, et al. Thrombospondin-1 restrains neutrophil granule serine protease function and regulates the innate immune response during Klebsiella pneumoniae infection. Mucosal Immunol. 2015;8(4):896-905.

29. Cochrane CG, Spragg R, Revak SD. Pathogenesis of the adult respiratory distress syndrome. Evidence of oxidant activity in bronchoalveolar lavage fluid. J Clin Invest. 1983;71(3):754-761.

30. Lee CT, Fein AM, Lippmann M, Holtzman H, Kimbel P, Weinbaum G. Elastolytic activity in pulmonary lavage fluid from patients with adult respiratory-distress syndrome. N Engl J Med. 1981;304(4):192-196.

31. Wilkinson TS, et al. Ventilator-associated pneumonia is characterized by excessive release of neutrophil proteases in the lung. Chest. 2012;142(6):1425-1432.

32. Qin X, Zerr DM, McNutt MA, Berry JE, Burns JL, Kapur RP. Pseudomonas aeruginosa syntrophy in chronically colonized airways of cystic fibrosis patients. Antimicrob Agents Chemother. 2012;56(11):5971-5981.

33. Silverstein RL. Platelet CD36 links not only $\alpha$-granule-derived proteins to thrombus stability but also metabolic and oxidant stress to a prothrombotic phenotype. Arterioscler Thromb Vasc Biol. 2014;34(6):1120-1121.

34. Johnson DA, Carter-Hamm B, Dralle WM. Inactivation of human bronchial mucosal proteinase inhibitor by Pseudomonas aeruginosa elastase. Am Rev Respir Dis. 1982;126(6):1070-1073.

35. Guyot N, et al. Functional study of elafin cleaved by Pseudomonas aeruginosa metalloproteinases. Biol Chem. 2010;391(6):705-716.

36. Hogg PJ, Owensby DA, Chesterman CN. Thrombospondin 1 is a tight-binding competitive inhibitor of neutrophil cathepsin G. Determination of the kinetic mechanism of inhibition and localization of cathepsin $\mathrm{G}$ binding to the thrombospondin 1 type 3 repeats. J Biol Chem. 1993;268(29):21811-21818.

37. Hogg PJ, Owensby DA, Mosher DF, Misenheimer TM, Chesterman CN. Thrombospondin is a tight-binding competitive inhibitor of neutrophil elastase. J Biol Chem. 1993;268(10):7139-7146.

38. Filloux A. Protein secretion systems in Pseudomonas aeruginosa: an essay on diversity, evolution, and function. Front Microbiol. 2011;2:155

39. Miyata S, Casey M, Frank DW, Ausubel FM, Drenkard E. Use of the Galleria mellonella caterpillar as a model host to study the role of the type III secretion system in Pseudomonas aeruginosa pathogenesis. Infect Immun. 2003;71(5):2404-2413.

40. Ryu H, Choi K, Qu Y, Kwon T, Lee JS, Han J. Patient-derived airway secretion dissociation technique to isolate and concentrate immune cells using closed-loop inertial microfluidics. Anal Chem. 2017;89(10):5549-5556.

41. Hogg PJ, Jiménez BM, Chesterman CN. Identification of possible inhibitory reactive centers in thrombospondin 1 that may bind cathepsin G and neutrophil elastase. Biochemistry. 1994;33(21):6531-6537.

42. Hogg PJ, Stenflo J, Mosher DF. Thrombospondin is a slow tight-binding inhibitor of plasmin. Biochemistry. 1992;31(1):265-269.

43. Lu SM, et al. Predicting the reactivity of proteins from their sequence alone: Kazal family of protein inhibitors of serine proteinases. Proc Natl Acad Sci USA. 2001;98(4):1410-1415.

44. Rastinejad F, Polverini PJ, Bouck NP. Regulation of the activity of a new inhibitor of angiogenesis by a cancer suppressor gene. Cell. 1989;56(3):345-355.

45. Vuorio E, de Crombrugghe B. The family of collagen genes. Annu Rev Biochem. 1990;59:837-872.

46. Lawler J, Hynes RO. The structure of human thrombospondin, an adhesive glycoprotein with multiple calcium-binding sites and homologies with several different proteins. J Cell Biol. 1986;103(5):1635-1648.

47. Bein K, Simons M. Thrombospondin type 1 repeats interact with matrix metalloproteinase 2. Regulation of metalloproteinase activity. J Biol Chem. 2000;275(41):32167-32173.

48. Bornstein P. Thrombospondins: structure and regulation of expression. FASEB J. 1992;6(14):3290-3299.

49. Rodriguez-Manzaneque JC, Lane TF, Ortega MA, Hynes RO, Lawler J, Iruela-Arispe ML. Thrombospondin-1 suppresses spontaneous tumor growth and inhibits activation of matrix metalloproteinase- 9 and mobilization of vascular endothelial growth factor. Proc Natl Acad Sci USA. 2001;98(22):12485-12490.

50. Lawler J, et al. Thrombospondin-1 is required for normal murine pulmonary homeostasis and its absence causes pneumonia. J Clin Invest. 1998;101(5):982-992.

51. Sutterwala FS, Mijares LA, Li L, Ogura Y, Kazmierczak BI, Flavell RA. Immune recognition of Pseudomonas aeruginosa mediated by the IPAF/NLRC4 inflammasome. J Exp Med. 2007;204(13):3235-3245.

52. Cohen TS, Prince AS. Activation of inflammasome signaling mediates pathology of acute P. aeruginosa pneumonia. J Clin Invest. 2013;123(4):1630-1637. 
53. Franchi L, Stoolman J, Kanneganti TD, Verma A, Ramphal R, Núñez G. Critical role for Ipaf in Pseudomonas aeruginosainduced caspase-1 activation. Eur J Immunol. 2007;37(11):3030-3039.

54. Lightfield KL, et al. Critical function for Naip5 in inflammasome activation by a conserved carboxy-terminal domain of flagellin. Nat Immunol. 2008;9(10):1171-1178.

55. Azghani AO. Pseudomonas aeruginosa and epithelial permeability: role of virulence factors elastase and exotoxin A. Am $J$ Respir Cell Mol Biol. 1996;15(1):132-140.

56. Yang J, et al. Mechanistic insights into elastin degradation by pseudolysin, the major virulence factor of the opportunistic pathogen Pseudomonas aeruginosa. Sci Rep. 2015;5:9936.

57. Bejarano PA, Langeveld JP, Hudson BG, Noelken ME. Degradation of basement membranes by Pseudomonas aeruginosa elastase. Infect Immun. 1989;57(12):3783-3787.

58. Wang T, et al. Thrombocytopenia is associated with acute respiratory distress syndrome mortality: an international study. $P L o S$ ONE. 2014;9(4):e94124.

59. Singer M, et al. The third international consensus definitions for sepsis and septic shock (Sepsis-3). JAMA. 2016;315(8):801-810

60. Bhandari V, et al. Hyperoxia causes angiopoietin 2-mediated acute lung injury and necrotic cell death. Nat Med. 2006;12(11):1286-1293.

61. Deatherage DE, Barrick JE. Identification of mutations in laboratory-evolved microbes from next-generation sequencing data using breseq. Methods Mol Biol. 2014;1151:165-188.

62. Liberati NT, et al. An ordered, nonredundant library of Pseudomonas aeruginosa strain PA14 transposon insertion mutants. Proc Natl Acad Sci USA. 2006;103(8):2833-2838.

63. Shanks RM, Caiazza NC, Hinsa SM, Toutain CM, O'Toole GA. Saccharomyces cerevisiae-based molecular tool kit for manipulation of genes from gram-negative bacteria. Appl Environ Microbiol. 2006;72(7):5027-5036.

64. Lee JS, et al. TLR-4 pathway mediates the inflammatory response but not bacterial elimination in E. coli pneumonia. Am J Physiol Lung Cell Mol Physiol. 2005;289(5):L731-L738.

65. Ishii T, et al. Neutrophil elastase contributes to acute lung injury induced by bilateral nephrectomy. Am J Pathol. 2010;177(4):1665-1673.

66. Truss NJ, Armstrong PC, Liverani E, Vojnovic I, Warner TD. Heparin but not citrate anticoagulation of blood preserves platelet function for prolonged periods. J Thromb Haemost. 2009;7(11):1897-1905.

67. Merritt TA, et al. Elastase and alpha 1-proteinase inhibitor activity in tracheal aspirates during respiratory distress syndrome Role of inflammation in the pathogenesis of bronchopulmonary dysplasia. J Clin Invest. 1983;72(2):656-666. 